\title{
Tocilizumab-Induced Sweet Syndrome in a Patient With Polymyalgia Rheumatica
}

\author{
Federica Filippi, ${ }^{1}$ Marco A. Chessa, ${ }^{1}$ Annalisa Patrizi, ${ }^{1}$ Carlotta Baraldi, ${ }^{1}$ \\ Francesca Ferrara, ${ }^{1}$ Federico Bardazzi ${ }^{1}$
}

1 Dermatology, Department of Experimental, Diagnostic and Specialty Medicine, University of Bologna, Italy

Key words: tocilizumab, tocilizumab-induced Sweet syndrome, drug-induced Sweet syndrome, neutrophilic dermatosis

Citation: Filippi F, Chessa MA, Patrizi A, Baraldi C, Ferrara F, Bardazzi F. Tocilizumab-induced Sweet syndrome in a patient with polymyalgia rheumatica. Dermatol Pract Concept. 2020;10(1):e2020019. DOI: https://doi.org/10.5826/dpc.1001a19

Accepted: July 23, 2019; Published: December 31, 2019

Copyright: (02019 Filippi et al. This is an open-access article distributed under the terms of the Creative Commons Attribution License, which permits unrestricted use, distribution, and reproduction in any medium, provided the original author and source are credited.

Funding: None.

Competing interests: The authors have no conflicts of interest to disclose.

Authorship: F.F., M.A.C., and F.B. contributed equally to this work. All authors have contributed significantly to this publication.

Corresponding author: Marco A. Chessa, MD, Dermatology, Department of Experimental, Diagnostic and Specialty Medicine, University of Bologna, Via Massarenti, 1-40138 Bologna, Italy. Email: marcoadriano.chessa@gmail.com

\section{Introduction}

Tocilizumab (TCZ) is a humanized monoclonal antibody directed against the interleukin 6 receptor, which has a main use in the treatment of several rheumatological diseases but also giant cell arteritis and polymyalgia rheumatica. Despite the undoubted efficacy of this target therapy, immuno-mediated adverse events have increasingly been reported, including cutaneous sarcoidosis, psoriasis, acute generalized exanthematous pustulosis, leukocytoclastic vasculitis, drug reaction with eosinophilia and systemic symptoms (DRESS) syndrome, and granuloma annulare.

\section{Case Presentation}

A 75-year-old man was referred to our clinic 5 days after the onset of diffuse multiple painful targetoid plaques asymmetrically distributed on the lower extremities, trunk, neck, and nucha. The lesions were starting to coalesce to form irregular, sharply bordered plaques. He also presented with a marked periocular swelling (Figure 1, A-D).
The patient had a history of polymyalgia rheumatica and had been treated with oral steroids for 10 years, which were then suspended because of the onset of severe osteoporosis, with recurrence of symptoms. Hence, subcutaneous TCZ was started at the dosage of $162 \mathrm{mg} /$ week. Four days after the first administration of TCZ, the patient reported sporadic painful annular plaques on his neck; the day after the second injection, the lesions were spreading to the rest of the body. Moreover, the patient reported arthralgias, general malaise, headache, and fever $\left(38.3^{\circ} \mathrm{C}\right)$.

Clinical observations and laboratory values were evaluated to rule out other drug-induced conditions with cutaneous involvement. Investigations yielded a peripheral leukocytosis with neutrophilia and elevation of erythrocyte sedimentation rate and C-reactive protein. In erythema multiforme the lesions are itchy and typically start on the extremities symmetrically, with centripetal spreading [1]. A key characteristic of urticaria-angioedema is the evanescence of skin lesions (wheals last less than 24 hours), whereas our patient presented with persistent plaques. Autoimmune screen was negative and sun-exposed areas were spared, so drug-induced 
Figure 1. Clinical and histopathological findings. (A-D) Multiple painful targetoid plaques characterized by irregular sharp border, central desquamative rim in some lesions and asymmetrically distributed on lower extremities, trunk, neck, and nucha. Periocular swelling, left side worse than right. (E) Dense infiltrate composed largely of neutrophils in the upper dermis (H\&E, $\times 4)$. (F) Higher magnification of the neutrophilic infiltrate with some nuclear fragmentation; vasodilation and swelling of endothelium with moderate extravasation of erythrocytes and prominent edema were also present $(\mathrm{H} \& \mathrm{E}, \times 10)$. $(\mathrm{G}, \mathrm{H})$ Clinical remission within 3 months, after tocilizumab interruption and systemic and topical steroid administration.

subacute cutaneous lupus erythematosus (antinuclear antibody positive in $60 \%-80 \%$ of cases) was excluded. Finally, Wells syndrome is characterized by itchy and indurated plaques with erythema and edema, and systemic symptoms may be present, so histopathology is mandatory for differential diagnosis. Histopathological examination showed a dense perivascular infiltrate composed largely of neutrophils throughout the papillary dermis (Figure 1, E and F).

Sweet syndrome is the most representative entity of febrile neutrophilic dermatoses. It can present as 1 of 3 clinical types: classic or idiopathic, malignancy-associated, or drug-induced. In drug-induced Sweet syndrome, all of the following criteria should be present to achieve the diagnosis: (1) abrupt onset of painful erythematous plaques or nodules; (2) histopathological findings of dense neutrophilic infiltrate without evidence of leukocytoclastic vasculitis; (3) fever $>38^{\circ} \mathrm{C}$; (4) temporal relation between use of medication and clinical presentation or relapse with readministration; and (5) disappearance of lesions after drug discontinuation or treatment with systemic corticosteroids [2]. Our case fulfilled all of these criteria and thus the diagnosis of TCZ-induced Sweet syndrome was made.

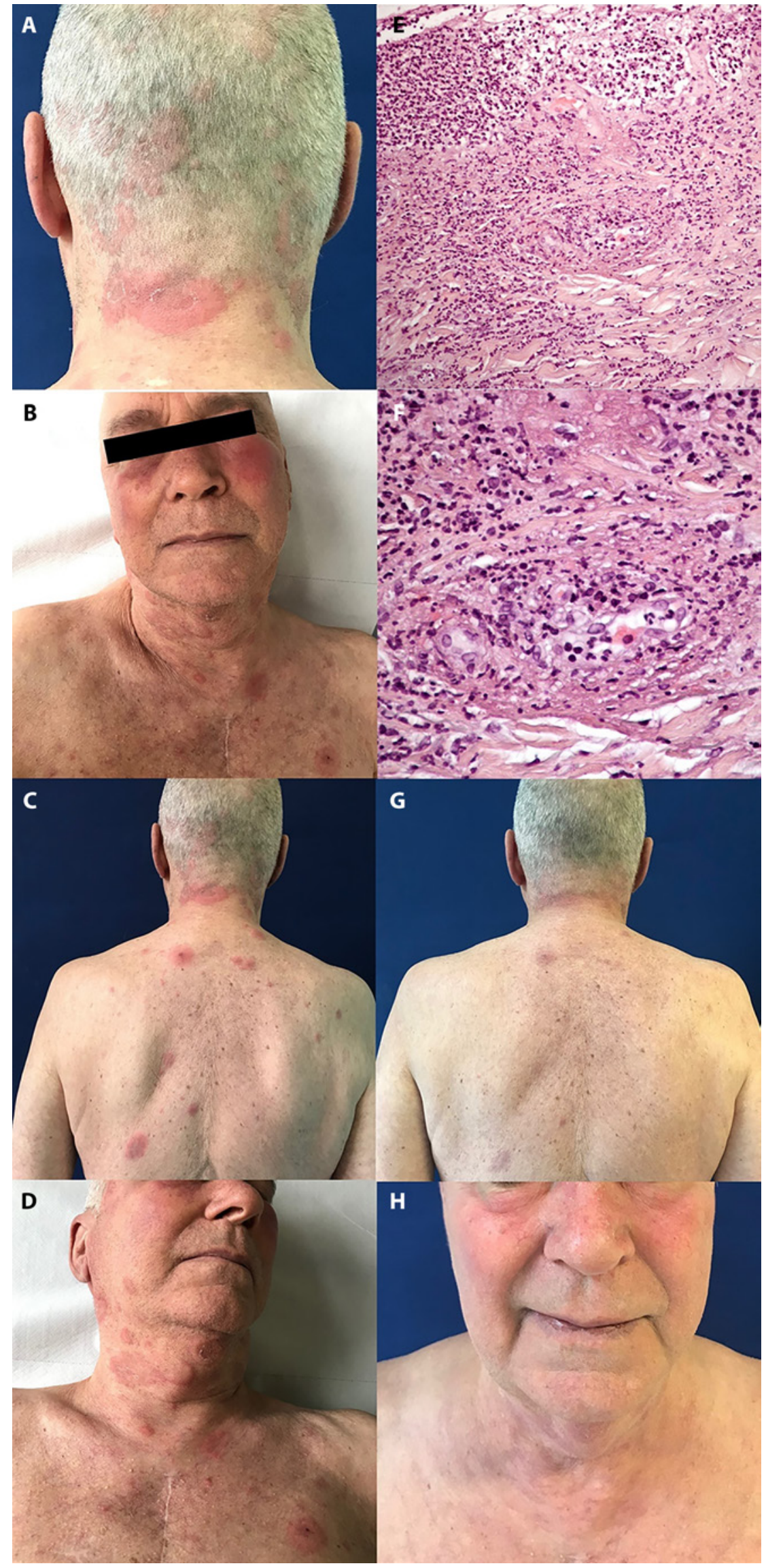

Consequently, TCZ was discontinued and oral prednisone at a dosage of $0.6 \mathrm{mg} / \mathrm{kg} /$ day and a topical steroid
( $0.05 \%$ clobetasol propionate cream) once daily were prescribed. The systemic steroid was tapered to $10 \mathrm{mg} /$ day within 
6 weeks. The patient improved significantly after 4 weeks and had a complete remission in 3 months (Figure 1, G and $\mathrm{H})$. Successively, to reduce the corticosteroid intake, in cooperation with his rheumatologist, an off-label treatment with adalimumab (40 mg every 2 weeks subcutaneously) was started. After a 3-month follow-up, no relapse was observed.

\section{Conclusions}

In conclusion, clinicians should be aware of this possible reaction even though the causality has not been proven yet in the literature.

\section{References}

1. Baroni A, Piccolo V, Russo T, Cozzolino D, Mascolo M, Chessa MA. Norfloxacin-in- duced subacute cutaneous lupus with erythema multiforme-like lesions: the enigma of the Rowell syndrome.J Dtsch Dermatol Ges. 2014;12(11):1039-1042.

2. Walker DC, Cohen PR. Trimethoprim-sulfamethoxazole-associated acute febrile neutrophilic dermatosis: case report and review of drug-induced Sweet's syndrome. J Am Acad Dermatol. 1996;34(5 Pt 2):918-923. 